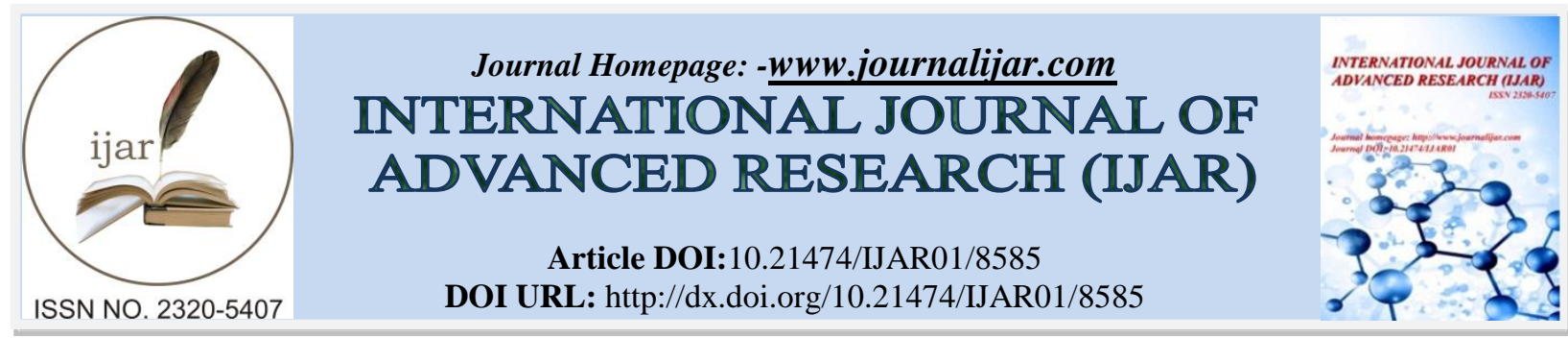

RESEARCH ARTICLE

\title{
A CASE OF OBSTRUCTIVE SLEEP APNOEA.
}

Papaiah Nirmal Pudota ${ }^{1}$ and N.h.noorul ameen ${ }^{2}$. Department of General Medicine, Sree Balaji Medical College and Hospital, India.

\section{Manuscript Info}

\section{Manuscript History}

Received: 14 December 2018

Final Accepted: 16 January 2019

Published: February 2019

Key words:-

Behaviour, determinant, factor, health, seeking.

\begin{abstract}
Health is not only determined by biological factor but also by both cultural and social environment. So the social determinants are very important factor of health. The social determinants of health are the conditions in which people are "born, grow, live, work, age," and which shape their health status. The main objective of this study was to know the affecting factors and social determinant factors of health seeking behaviour in the study area and to know the people's perception regarding health and disease. The researcher used such as interview schedule (face to face interview technique using semi structure questions), case study. In this study the researcher has used the survey method for selection of the respondent. The finding reveals that folk tradition and culture mostly close to the health seeking behaviour of the people. Cultural influence was major factor of health among the people. Culture shapes the people what to do, where to go etc. regarding the illness treatment. Therefore, in the process of health seeking behaviour the people influence from the factor of geography, availability, education, income, poverty, gender disparity, social support and network, we feelings, interaction, taking of decision, social ties, share of information, emotion, togetherness of activity and integration of this whole activity etc.
\end{abstract}

Copy Right, IJAR, 2019,. All rights reserved.

\section{Introduction:-}

Adrenal hemorrhage is a life threatening condition that can present with non specific symptoms such as abdominal pain, nausea,vomiting, fatigue.Rarely adrenal hemorrhage can be picked up as in incidental finding on imaging,without any symptoms.Examination findings are fever(most common),Tachycardia,Skin hyperpigmentation and shock in severe bilateral adrenal hemorrhage.

Most cases are caused by acute,Stressful illness(example,Infection,ACS,Heart failure, Waterhouse Friedrichson Syndrome-Meningococcal septicemia.)Other causes include Blunt trauma,Thromboembolic diseases like APLA,Anticoagulant use,Thrombocytopenia,Pregnancy complications, TB amyloidosis, and Metastatic tumours.A muticenter Case controlled study was done to study the risk factors associated with adrenal hemorrhage.Thrombocytopenia,Heparin use,Sepsis were identified as major risk factors.

Corresponding Author:-Papaiah Nirmal Pudota.

Address:-Department of General Medicine, Sree Balaji Medical College and Hospital, India. 


\section{Report of the case:}

A 57 years old female patient who is a known case of hypertension on treatment presented with complaints of abdominal pain since 1 week which is dull aching type of pain,non localized.She also complains of multiple episodes of vomiting,non bilious,non projectile,containing food particles for 1 week.No h/o fever or burning micturition.No h/o any substance abuse.No h/o anticoagulant use,trauma,tuberculosis

On examination patient was conscious, oriented to time,place and person,vitals stable and afebrile.No skin hyperpigmentation or rashes.Per abdomen-there was no organomegaly,Epigastric tenderness was present.CNS examination revealed no focal neurologic defects.Labs done showed sodium 110,potassium 4.2, Chloride 79 ,Bicarbonate 21 ,Serum osmolality 223 , Urine spot sodium 100 ,Serum cortisol was 1.85 .Echo done showed no RWMA,EF 64\%.USG abdomen showed B/l Hydronephrosis after which CT whole abdomen is done which showed Splenomegaly and B/L adrenal hemorrhage.CT thorax showed minimal left pleural effusion.Urine culture showed klebsiella.A diagnosis of $\mathrm{B} / \mathrm{L}$ adrenal hemorrhage due to urosepsis was made and managed in ICU.APLA workup done which was negative.ANA/DSDNA was negative.Patient was treated with inj.Meropenem $1 \mathrm{~g}$ BD,Inj hydrocortisone, and other supportive measures in ICU.Patient improved symptomatically and shifted to ward.Patient was discharged with T.Hisone $10 \mathrm{mg} 1-0-1 / 2$ and was advised to follow up

\section{Discussion:-}

$\mathrm{B} / \mathrm{l}$ adrenal hemorrhage can be caused by a number of conditions .In our patient,the underlying cause was UROSEPSIS.The underlying mechanism in a non traumatic adrenal hemorrhage is unclear.Adrenal gland has a poor venous drainage,but the arterial supply is rich.During stress, there is increased ACTH secretion which causes increased arterial blood flow exceeding the venous drainage capacity causing adrenal hemorrhage.Adrenal vein thrombosis is another proposed mechanism which can occur in APLA,SEPSIS,HEPARIN induced THROMBOCYTOPENIA.For the harmone deficiency to be clinically evident,90\% of the adrenal tissue must be destroyed.Hence minor adrenal hemorrhage might be unnoticed.

$\mathrm{CT}$ abdomen is the investigation of choice in Adrenal hemorrhage.HYponatremia,Hyperkalemia, and Hypoglycemia are present in most cases,However their absence does not exclude the diagnosis.High ACTH and low cortisol is diagnostic of Primary adrenal Insufficiency.

Acute adrenal insuffiency is a medical emergency.Supportive care is essential which includes fluid and electrolyte correction,monitor and stabilize blood pressure.,blood transfusion in cases of severe hemorrhage.If acute adrenal insuffiency is clinically suspected,Hydrocortisone should be ggiven without delay(100 $\mathrm{mg}$ bolus injection,followed by $200 \mathrm{mg}$ per $24 \mathrm{hr}$ either as continuous infusion or $50 \mathrm{mg}$ every $6 \mathrm{hr}$., aling with intra venous fluid resuscitation,after withdrawing samples for cortisol assay without waiting for results.Treat the underlying disease.On discharge, Parenteral glucocorticoids can be converted to oral.

\section{Conclusion:-}

Adrenal Hemorrhage is a serious condition which can present with non specific signs and symptoms,hence prompt diagnosis and treatment is required.It could be due to number of causes.In our Patient the underlying etiology was urosepsis. 